\title{
An optimized approach to germ-free rearing in the jewel wasp Nasonia
}

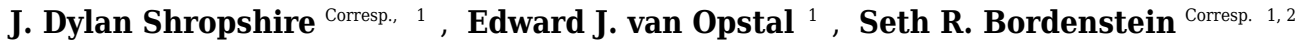 \\ 1 Biological Sciences, Vanderbilt University, Nashville, TN, United States \\ 2 Pathology, Microbiology \& Immunology, Vanderbilt University, Nashville, TN, United States \\ Corresponding Authors: J. Dylan Shropshire, Seth R. Bordenstein \\ Email address: dylan.shropshire@vanderbilt.edu, s.bordenstein@vanderbilt.edu
}

Development of a Nasonia in vitro germ-free rearing system in 2012 enabled investigation of Nasonia-microbiota interactions and real-time visualization of parasitoid metamorphosis. However, the use of antibiotics, bleach, and fetal bovine serum introduced artifacts relative to conventional rearing of Nasonia. Here, we optimize the germ-free rearing procedure by using filter sterilization in lieu of antibiotics and by removing residual bleach and fetal bovine serum. Comparison of these methods reveals no influence on larval survival or growth, and a $52 \%$ improvement in adult production. Additionally, adult males produced in the new germ-free system are similar in size to conventionally reared males. Experimental implications of these changes are discussed. 


\section{An Optimized Approach to Germ-free Rearing in the Jewel Wasp Nasonia}

2 J. Dylan Shropshire ${ }^{1 *}$, Edward J. van Opstal ${ }^{1 *}$, and Seth R. Bordenstein ${ }^{1,2}$

$3 \quad{ }^{1}$ Vanderbilt University, Department of Biological Sciences, Nashville, TN, USA

4 2Vanderbilt University, Department of Pathology, Microbiology, and Immunology, Nashville,

5 TN, USA

$6 \quad *$ These authors contributed equally to this work

7

8 Corresponding Authors:

9 J. Dylan Shropshire

10 U7217 BSB / MRB III, $46521^{\text {st }}$ Ave South, Nashville, TN, 37232, USA

11 Email address: dylan.shropshire@,vanderbilt.edu

12 Seth R. Bordenstein

13 U7215 BSB / MRB III, $46521^{\text {st }}$ Ave South, Nashville, TN, 37232, USA

14 Email Address: s.bordenstein@vanderbilt.edu 


\section{ABSTRACT}

17 Development of a Nasonia in vitro germ-free rearing system in 2012 enabled investigation of

18 Nasonia-microbiota interactions and real-time visualization of parasitoid metamorphosis.

19 However, the use of antibiotics, bleach, and fetal bovine serum introduced artifacts relative to conventional rearing of Nasonia. Here, we optimize the germ-free rearing procedure by using

21 filter sterilization in lieu of antibiotics and by removing residual bleach and fetal bovine serum.

22 Comparison of these methods reveals no influence on larval survival or growth, and a 52\%

23 improvement in adult production. Additionally, adult males produced in the new germ-free

24 system are similar in size to conventionally reared males. Experimental implications of these

25 changes are discussed. 


\section{INTRODUCTION}

The Nasonia genus (Ashmead \& Smith, 1904) consists of four closely related interfertile parasitoid wasp species and has been a powerful model for the study of genetics (Davies \& Tauber, 2015; Lynch, 2015; Raychoudhury et al., 2010), evolution (Bordenstein, O'Hara \& Werren, 2001; Bordenstein \& Werren, 2007; Brucker \& Bordenstein, 2013; Clark et al., 2010), endosymbiosis (Bordenstein, O'Hara \& Werren, 2001; Ferree et al., 2008), development (Rivers \& Losinger, 2014; Verhulst et al., 2013; Zwier et al., 2012), behavior (Baeder \& King, 2004; Beukeboom \& van den Assem, 2001; Clark et al., 2010; Drapeau \& Werren, 1999; Raychoudhury et al., 2010), pheromonal communication (Diao et al., 2016; Ruther \& Hammerl, 2014; Steiner, Hermann \& Ruther, 2006), and other areas. The design and publication of an in vitro system for Nasonia in 2012 detached Nasonia from its fly host, allowed for real-time monitoring of development, and provided an avenue to study how microbes influence Nasonia biology (Brucker \& Bordenstein, 2012a). These tools advanced the Nasonia system to explore how gut microbiota influence development and hybrid lethality (Brucker \& Bordenstein, 2013).

Nasonia germ-free rearing involves two major components: (i) sterilizing Nasonia embryos and (ii) providing larvae with sterilized food in an in vitro system. Embryo sterilization is conducted by picking Nasonia embryos from pupal fly hosts (typically Sarcophaga bullata; Werren \& Loehlin, 2009a) and then rinsing the embryos with bleach followed by sterile water (Brucker \& Bordenstein, 2012a). Producing Nasonia Rearing Medium (NRM) involves the collection of hundreds of fly pupae, extraction of proteinaceous fluids from those pupae, addition of fetal bovine serum (FBS) and Schneider's Drosophila medium for additional nutrition, filter sterilization, and addition of antibiotics (Fig 1; Brucker \& Bordenstein, 2012a). Sterilized 
49

50

51

52

53

54

55

56

57

58

59

60

61

62

63

64

65

66

67

68

69

70

embryos are then placed on a transwell permeable membrane with filter-sterilized NRM underneath for feeding (Brucker \& Bordenstein, 2012a).

This protocol yielded similarly sized Nasonia to those from in vivo rearing (Brucker \& Bordenstein, 2012a). However, NRM production relies on introducing foreign and potentially harmful elements such as bleach, FBS, and antibiotics. Removal of each component carries its own rationale. For example, the bleach treatment was intended to kill surface bacteria on the puparium of host flies and remove particulates (Brucker \& Bordenstein, 2012a). However, surface bacteria will be removed during filtration and residual bleach from the rinse may persist in the final NRM product as a toxic agent. Furthermore, FBS is added as a nutritional supplement to increase larval survival and development (Brucker \& Bordenstein, 2012a), but Nasonia do not frequently encounter components of FBS including bovine-derived hormones such as testosterone, progesterone, insulin, and growth hormones (Honn, Singly \& Chavin, 1975).

Finally, antibiotics are a confounding variable and removing them will provide more flexibility to bacterial inoculations in the in vitro system.

This study removes these three major components of the original NRM and optimizes the procedure by eliminating extraneous steps and utilizing quicker approaches. These changes are validated by directly comparing germ-free Nasonia reared on either the original (NRMv1) or optimized (NRMv2) media for larval and pupal survival, larval growth, and adult production.

7 The morphology of adults produced both in vitro and in vivo is then compared.

MATERIALS AND METHODS

Nasonia rearing medium (NRMv1) 

2009a). Approximately $150 \mathrm{ml}$ of $S$. bullata pupae were transferred to a sterile $250 \mathrm{ml}$ beaker after close inspection to remove larvae, poor quality pupae, and debris. A solution of $10 \%$ Clorox bleach was then added to the beaker to cover the pupae. After five minutes, the bleach was drained from the beaker and the pupae were repeatedly rinsed with sterile millipore water until the scent of bleach was absent. Sterile millipore water was added in the beaker to approximately $2 / 3$ the volume of pupae, covered, and placed in a $36^{\circ} \mathrm{C}$ water bath to soften the puparium. $S$. bullata pupae were homogenized using a household kitchen blender and filtered through a 100 $\mu \mathrm{m}$ cell strainer (Fisherbrand, Thermo Fisher scientific Incorporated, Waltham, MA). The filtrate was poured evenly across two $50 \mathrm{ml}$ conical tubes (Falcon, Corning Incorporated, Corning, NY) and centrifuged at $4{ }^{\circ} \mathrm{C}(25,000 \times \mathrm{xG})$ for 5 minutes to separate the sediment, protein, and lipid layers, and a 22 gauge needle (BD PrecisionGlide, Becton, Dickinson and Company, Franklin Lakes, NJ) was used to remove the protein layer. The protein layer was combined with $50 \mathrm{ml}$ of product was stored at $4^{\circ} \mathrm{C}$ until use (Fig. 1). 
Approximately the same number of $S$. bullata pupae were collected as described above.

94 Pupae were subsequently rinsed in sterile millipore water to remove small particulates. They

95 were then crushed by hand through a $100 \mu \mathrm{m}$ nylon net (EMD Millipore, Merck Millipore, Billerica, MA) and the filtrate was collected in a sterile $250 \mathrm{ml}$ glass beaker. Nylon powder-free non-sterile gloves were worn during this extraction. The filtrate was centrifuged at $4^{\circ} \mathrm{C}$ $(25,000 \mathrm{xG})$ for 10 minutes to separate the sediment, protein, and lipid layers. Using a 22 gauge needle (BD PrecisionGlide, Becton, Dickinson and Company, Franklin Lakes, NJ), the protein layer was transferred to a sterile beaker. Schneider's Drosophila media was added to the protein extract to triple the volume and, using a reusable vacuum filtration apparatus (Nalgene, Thermo Fisher scientific Incorporated, Waltham, MA), the resulting mixture was passed through filters with gradually smaller pore sizes $(11,6,2.5,0.8$, and $0.45 \mu \mathrm{m})$. A $0.22 \mu \mathrm{m}$ syringe filter (Costar, Corning Incorporated, Corning, NY) was used to remove bacteria. The final product was stored at $4^{\circ} \mathrm{C}$ until use (Fig. 1). The following is a step-wise protocol for the production of NRMv2.

1. Fill a sterilized beaker with $150 \mathrm{ml}$ of $S$. bullata pupa. Remove larvae, poor quality pupae, and debris.

2. In the beaker, cover pupae with sterile Millipore water, allow to sit for $1 \mathrm{~min}$, and strain to remove surface particulates from the puparium surface. Some moisture will remain on the pupae.

3. Crush the pupae by hand (covered with powder-free nitrile gloves) and squeeze juices through a $100 \mu \mathrm{m}$ nylon mesh to remove the $S$. bullata puparium.

4. Separate juices (approximately $70-90 \mathrm{ml}$ ) evenly into two $50 \mathrm{ml}$ conical tubes and seal tightly. 
115

5. Centrifuge the mixture for $10 \mathrm{~min}$ at $4^{\circ} \mathrm{C}(25,000 \mathrm{xG})$. The mixture will separate into three distinct layers: a sediment, protein, and lipid layer from bottom to top, respectively.

6. To prevent clogging during filtration, extract the protein layer using a 22 gauge sterile needle and transfer it to a sterile beaker under sterile laminar flow.

7. Add a 2:1 ratio of Schneider's Drosophila medium to the protein extract.

8. Using a vacuum filtration system, filter the media through progressively smaller pore sizes $(11,6,2.5,0.8$, and $0.45 \mu \mathrm{m}$ filters $)$ to remove increasingly smaller particulates. To prevent clogging, replace filter paper when flow begins to slow.

9. Sterilize the media by filtering through a $0.22 \mu \mathrm{m}$ syringe filter, taking care to use aseptic technique.

10. Store at $4^{\circ} \mathrm{C}$ for up to 2 weeks.

11. Filter NRM through a $0.22 \mu \mathrm{m}$ syringe filter before use to ensure sterility and remove sedimentation.

\section{Nasonia strains and collections}

N. vitripennis (strain AsymCx; Wolbachia uninfected) mated females were hosted on $S$.

bullata pupae and housed in glass culture tubes capped with cotton at $25 \pm 2^{\circ} \mathrm{C}$ in constant light, as previously described (Werren \& Loehlin, 2009b). After 10-12 days, S. bullata pupariums were opened and virgin $N$. vitripennis females were collected as pupae from the resulting offspring. Upon adult eclosion, individual virgin females were isolated and provided two S. bullata pupae for hosting to increase the number of eggs deposited in subsequent hostings. In haplodiploids, virgin females are fecund and lay all male (haploid) offspring. Two days after initial hostings, 
137 females were provided with a new S. bullata pupae housed in a Styrofoam plug, allowing her to

138 oviposit only on the anterior end of the host for easy embryo collection. polyester membrane (Costar, Corning Incorporated, Corning, NY) and sterilized twice with $70 \mu \mathrm{l}$ $10 \%$ bleach solution and once with $70 \mu 170 \%$ ethanol solution. The embryos were then rinsed

145 three times with $80 \mu \mathrm{l}$ sterile millipore water. After rinsing, the transwell insert was moved into a 14624 well plate with $250 \mu \mathrm{l}$ of NRM in the well. All plates were stored in a sterile Tupperware box 147 at $25 \pm 2^{\circ} \mathrm{C}$ in constant light conditions for the duration of the experiment. Under sterile laminar 148 flow, transwells were moved to new wells with $250 \mu \mathrm{l}$ of fresh NRM every second day. Approximately $1.5 \mathrm{ml}$ of NRM was used per transwell over the duration of the experiment. After

150 eleven days, the transwells were moved to dry wells in a clean plate and the 12 empty

151 surrounding wells were filled with $1 \mathrm{ml}$ of sterile millipore water to increase humidity. Two 152 plates with 12 transwells each (total of 24) were set up using either NRMv1 or NRMv2 by JDS 153 for Nasonia in vitro rearing in May 2016. Replicate rearing and collection of larval and pupal 154 survival data was conducted on both NRMv1 ( $=9$ inserts) and NRMv2 ( $=13$ inserts) by EVO 155 in April 2015 and March 2016 respectively (Fig. S1). 
microscope-attached AmScope MT1000 camera. A baseline for the number of larvae present in a well was determined by counting the number of larvae present in transwell pictures 3 days after embryo deposition on the transwell membranes (Day 3). Survival estimates were determined by counting the number of live larvae on Day 6 and the number of live larvae and pupae on Day 14, compared to Day 3. Larvae and pupae were identified as dead if they were visibly desiccated or malformed. Larval length was determined using ImageJ software by measuring the anterior to posterior end of larvae on Days 3, 6, and 14. The proportion of adults produced by a transwell was determined as follows: (the number of larvae on Day 3 - the number of dead larvae and pupae remaining on Day 20) $\div$ the number of larvae on Day 3. Pictures of conventionally reared and germ-free (NRMv2) adult males were taken, and ImageJ was used to measure head width, which is a correlate for body size in Nasonia (Blaul \& Ruther, 2012; Tsai et al., 2014).

\section{RESULTS} conventionally reared $N$. vitripennis and there were no differences in larval survival or larval growth over development (Brucker \& Bordenstein, 2012a). Here we demonstrate, in comparisons between NRMv1 and NRMv2, that there is also no difference in larval and pupal survival to Day 14 (Fig 2A; Mann-Whitney U (MWU) for Day $6 \mathrm{p}=0.19$ and Day $14 \mathrm{p}=0.41$ ) nor length, measured as the distance from the anterior to posterior end (Fig 2B; MWU for Day 3 $\mathrm{p}=0.26$, Day $6 \mathrm{p}=0.18$, Day $14 \mathrm{p}=0.13$ ). A replicate experiment reveals that the survival results are repeatable (Fig S1; MWU for Day $6 \mathrm{p}=0.23$ and Day $14 \mathrm{p}=0.06$ ). Moreover, a visual comparison of larval sizes on NRMv1 and NRMv2 shows no major differences (Fig 2C-F). 
181 These findings indicate that removal of residual bleach, FBS, and antibiotics does not have a

182 significant impact on larval survival or development.

NRMv1 yielded low adult survival compared to conventional rearing (Brucker \&

184 Bordenstein, 2012a). To investigate if using NRMv2 improves larval to adult survival, both the

185 number of transwells producing adults and the average number of adults produced per transwell

186 were compared between NRMv1 and NRMv2. The number of transwells that produced at least a

187 single adult did not differ between NRMv1 (79\% productive; N=24) and NRMv2 (88\%

188 productive; N=24; Fig 3A; Fisher's exact test $\mathrm{p}=0.7$ ). However and importantly, NRMv2

189 yielded a higher proportion of adults than NRMv1 (Fig 3B; MWU $p=0.001$ ), accounting for a

$19052 \%$ increase in larval to adult survival. Finally, to ensure that adults produced in the in vitro

191 system are similar in size to conventional adults, the head width of adult males produced on

$192 \mathrm{NRMv} 2(\mathrm{~N}=16)$ was compared to conventionally reared $(\mathrm{N}=16)$ adult males, and there was no

193 significant difference (Fig 3C; MWU $\mathrm{p}=0.72$ ).

\section{DISCUSSION}

The previously established Nasonia in vitro germ-free rearing protocol (Brucker \&

Bordenstein, 2012a), which involved sterilizing embryos and feeding the larvae NRMv1, was

crucial for conducting experiments on Nasonia-microbiota interactions (Brucker \& Bordenstein,

2013). However, this initial version of the germ-free rearing system contained highly artificial

elements such as bleach rinsing, FBS, and antibiotics (Fig 1; NRMv2). Following removal of

201

these elements, we show that the alterations to the NRM did not influence larval and pupal

202 survival to day 14 in replicate experiments (Fig 2A; Fig S1) or larval growth (Fig 2B), but 
203 importantly resulted in a 52\% increase in larval to adult survival (Fig 3B). Moreover, the size of

204 adult males produced on NRMv2 and in vivo do not differ (Fig 3C), suggesting that both in vitro

205 and in vivo rearing produce morphologically similar adults.

206 Aside from making the Nasonia in vitro system more biologically relevant, the new

207 media has multiple experimental implications. For example, antibiotics are a confounding

208 variable with unknown consequences to Nasonia biology, and they can hinder inoculation

209 capabilities of the system by causing bacterial communities introduced to rapidly shift in

210 composition. Thus, removal of antibiotics in NRMv2 makes it easier to derive conclusions and

211 may provide more flexibility for inoculations in vitro, namely introduction of full microbial

212 communities derived from Nasonia species. This new system permits the introduction of both

213 autochthonous and allochthonous microbial communities, enabling investigations of the

214 functional relevance of host-specific microbial communities or microbial species. For example,

215 the Nasonia microbiota exhibits "phylosymbiosis", a pattern in which microbial community

216 relationships parallel the phylogenetic relationships of the host species (Brucker \& Bordenstein,

217 2012b; Brucker \& Bordenstein, 2013). Transplanting communities between species will test the

218 functional relevance of phylosymbiosis.

219 Furthermore, improved survival of larvae to adults on NRMv2 makes obtaining sample

220 sizes of adults and the measurement of adult phenomes (e.g., physiology, anatomy, and behavior)

221 more feasible. In this context, NRMv2 permits improved exploration of Nasonia adult-

222 microbiota interactions. For example, there are many examples of microbe-mediated signals used

223 in mate-choice, species recognition, and kin recognition (Reviewed in Shropshire \& Bordenstein,

224 2016). Nasonia species produce several different signals including cuticular hydrocarbons

225 (Buellesbach et al., 2013), abdominal sex pheromones (Diao et al., 2016), and cephalic 
226 pheromones housed in an oral gland (Miko \& Deans, 2014; Ruther \& Hammerl, 2014). This in

227 vitro rearing system allows for the exploration of the interaction of microbes with host signals to

228 test what role these complex interactions may have in adult behavior, insect communication, and

229 reproductive isolation.

230 Parasitoid wasps are also difficult to study developmentally because the fly host's

231 puparium obstructs visualization of the Nasonia larvae and pupae, preventing multiple measures

232 of a single individual over time. In vitro rearing of Nasonia allows for observations of single

233 individuals over developmental time and for strict control of larval diet, bacterial exposure, and

234 Nasonia density. Using this system, one may test how these variables influence metamorphosis

235 (Johnston \& Rolff, 2015), wing and body size (Rivers \& Losinger, 2014), craniofacial anomalies

236 (Werren et al., 2015), and many other physiological traits.

237 In summary, we streamlined and improved upon the Nasonia in vitro rearing system

238 while removing antibiotics and other factors from the equation. These changes open the door to

239 multidisciplinary studies of host-microbiota interactions and development and add to Nasonia's

240 utility as a model system. 

boxes indicate steps present in NRMv1 but are eliminated in NRMv2. Blue boxes indicate steps present in both procedures. Pictures to the right show the visual progression from $S$. bulla pupae to final NRM product. L, lipid layer; P, protein layer; S, sediment layer.

Figure 2. Comparison of Nasonia germ-free larval development on NRMv1 and

250

251

252

253

254

255

256

257

258

259

260

261

262

263

NRMv2. (A) The proportion of living Nasonia vitripennis in transwells on Days 3, 6, and 14. There are no statistically significant differences in larval and pupal survival to day 14 on NRMv1 and NRMv2. (B) Equivalent larval lengths measured from anterior to posterior end in mm. (C-F) Visual comparison of larvae reared on NRMv1 and NRMv2 on Days 6 and 9. Vertical bars with caps represent standard deviation from the mean.

Figure 3. Survival and size of Nasonia germ-free adult males. (A) Comparison of the number of transwells producing adults between NRMv1 and NRMv2. (B) Estimated proportion of adults eclosed in each transwell was determined as follows: (the number of larvae on Day 3 - the number of dead larvae and pupae remaining on Day 20$) \div$ the number of larvae on Day 3. (C) Adult head widths from germ-free males reared on NRMv2 and males reared conventionally. Adult eclosion was statistically different between the two media (Mann-Whitney U, P-value $=0.001$ ). All other measures were not significant with $\alpha=0.05$. Vertical bars with caps represent standard deviation from the mean. 

Figure S1. Replicate comparison of Nasonia germ-free larval development on NRMv1 and NRMv2. (A) The proportion of living Nasonia vitripennis in transwells on Days 3, 6, and 14. There are no statistically significant differences in larval and pupal survival to day 14 on NRMv1 and NRMv2. R1, replicate one conducted by JDS; R2, replicate two conducted by EVO. Vertical bars with caps represent standard deviation from the mean. 
273

274

275

276

277

278

279

280

281

282

283

284

285

286

287

288

289

290

291

292

293

294

295

296

297

298

299

300

301

302

303

304

305

306

307

308

309

310

311

312

313

314

315

316

317

\section{REFERENCES}

Ashmead WH, Smith HH. 1904. Classification of the Chalcid Flies: Or the Superfamily Chalcidoidea, with Descriptions of New Species in the Carnegie Museum, Collected in South America by Herbert $H$. Smith: authority of the Board of Trustees of the Carnegie Institute.

Baeder JM, King BH. 2004. Associative learning of color by males of the parasitoid wasp Nasonia vitripennis (Hymenoptera : Pteromalidae). Journal of Insect Behavior 17:201-213. DOI: 10.1023/B:JOIR.0000028570.93123.dc

Beukeboom LW, van den Assem J. 2001. Courtship and mating behaviour of interspecific Nasonia hybrids (Hymenoptera, Pteromalidae): a grandfather effect. Behavioral Genetics 31:167-177.

Blaul B, Ruther J. 2012. Body size influences male pheromone signals but not the outcome of mating contests in Nasonia vitripennis. Animal Behaviour 84:1557-1563. DOI: 10.1016/j.anbehav.2012.10.001

Bordenstein SR, O'Hara FP, Werren JH. 2001. Wolbachia-induced incompatibility precedes other hybrid incompatibilities in Nasonia. Nature 409:707-710. DOI: 10.1038/35055543

Bordenstein SR, Werren JH. 2007. Bidirectional incompatibility among divergent Wolbachia and incompatibility level differences among closely related Wolbachia in Nasonia. Heredity 99:278287. DOI: $10.1038 /$ sj.hdy.6800994

Brucker RM, Bordenstein SR. 2012a. In vitro cultivation of the hymenoptera genetic model, Nasonia. PLoS One 7:e51269. DOI: 10.1371/journal.pone.0051269

Brucker RM, Bordenstein SR. 2012b. Speciation by symbiosis. Trends in Ecology \& Evolution 27:443-451. DOI: 10.1016/j.tree.2012.03.011

Brucker RM, Bordenstein SR. 2013. The hologenomic basis of speciation: gut bacteria cause hybrid lethality in the genus Nasonia. Science 341:667-669. DOI: 10.1126/science.1240659

Buellesbach J, Gadau J, Beukeboom LW, Echinger F, Raychoudhury R, Werren JH, Schmitt T. 2013. Cuticular hydrocarbon divergence in the jewel wasp Nasonia: evolutionary shifts in chemical communication channels? Journal of Evolutionary Biology 26:2467-2478. DOI: 10.1111/jeb.12242

Clark ME, O'Hara FP, Chawla A, Werren JH. 2010. Behavioral and spermatogenic hybrid male breakdown in Nasonia. Heredity 104:289-301. DOI: 10.1038/hdy.2009.152

Davies NJ, Tauber E. 2015. WaspAtlas: a Nasonia vitripennis gene database and analysis platform. Database (Oxford) 2015. DOI: 10.1093/database/bav103

Diao W, Mousset M, Horsburgh GJ, Vermeulen CJ, Johannes F, van de Zande L, Ritchie MG, Schmitt T, Beukeboom LW. 2016. Quantitative Trait Locus Analysis of Mating Behavior and Male Sex Pheromones in Nasonia Wasps. G3: Genes/ Genomes/ Genetics. DOI: 10.1534/g3.116.029074

Drapeau MD, Werren JH. 1999. Differences in mating behaviour and sex ratio between three sibling species of Nasonia. Evolutionary Ecology Research 1:223-234.

Ferree PM, Avery A, Azpurua J, Wilkes T, Werren JH. 2008. A bacterium targets maternally inherited centrosomes to kill males in Nasonia. Current Biology 18:1409-1414. DOI: 10.1016/j.cub.2008.07.093

Honn KV, Singly JA, Chavin W. 1975. Fetal Bovine Serum: A multivariate standard. Proceedings of the socieity for experimental biology and medicine 149:344-347.

Johnston PR, Rolff J. 2015. Host and Symbiont Jointly Control Gut Microbiota during Complete Metamorphosis. PLoS Pathology 11:e1005246. 10.1371/journal.ppat.1005246

Lynch JA. 2015. The expanding genetic toolbox of the wasp Nasonia vitripennis and its relatives. Genetics 199:897-904. DOI: 10.1534/genetics.112.147512 
318

Miko I, Deans A. 2014. The mandibular gland in Nasonia vitripennis (Hymenoptera: Pteromalidae). bioRxiv. DOI: 10.1101/006569

Raychoudhury R, Desjardins CA, Buellesbach J, Loehlin DW, Grillenberger BK, Beukeboom L, Schmitt T, Werren JH. 2010. Behavioral and genetic characteristics of a new species of Nasonia. Heredity 104:278-288. 10.1038/hdy.2009.147

Rivers DB, Losinger M. 2014. Development of the gregarious ectoparasitoid Nasonia vitripennis using five species of necrophagous flies as hosts and at various developmental temperatures. Entomologia experimentalis et applicata 151:160-169. DOI: 10.1111/eea.12182

Ruther J, Hammerl T. 2014. An oral male courtship pheromone terminates the response of Nasonia vitripennis females to the male-produced sex attractant. Journal of Chemical Ecology 40:56-62. DOI: 10.1007/s10886-013-0372-2

Shropshire JD, Bordenstein SR. 2016. Speciation by Symbiosis: the Microbiome and Behavior. MBio 7. DOI: $10.1128 / \mathrm{mBio} .01785-15$

Steiner S, Hermann N, Ruther J. 2006. Characterization of a female-produced courtship pheromone in the parasitoid Nasonia vitripennis. Journal of Chemical Ecology 32:1687-1702. DOI: 10.1007/s10886-006-9102-3

Tsai Y-JJ, Barrows EM, Weiss MR, Zeh D. 2014. Pure Self-Assessment of Size During Male-Male Contests in the Parasitoid WaspNasonia vitripennis. Ethology 120:816-824. DOI: 10.1111/eth.12254

Verhulst EC, Lynch JA, Bopp D, Beukeboom LW, van de Zande L. 2013. A new component of the Nasonia sex determining cascade is maternally silenced and regulates transformer expression. PLoS One 8:e63618. DOI: 10.1371/journal.pone.0063618

Werren JH, Cohen LB, Gadau J, Ponce R, Baudry E, Lynch JA. 2015. Dissection of the complex genetic basis of craniofacial anomalies using haploid genetics and interspecies hybrids in Nasonia wasps. Developmental Biology. 10.1016/j.ydbio.2015.12.022

Werren JH, Loehlin DW. 2009a. Rearing Sarcophaga bullata fly hosts for Nasonia (parasitoid wasp). Cold Spring Harbor Protocols 2009:pdb prot5308. DOI: 10.1101/pdb.prot5308

Werren JH, Loehlin DW. 2009b. Strain maintenance of Nasonia vitripennis (parasitoid wasp). Cold Spring Harbor Protocols 2009:pdb prot5307. DOI: 10.1101/pdb.prot5307

Zwier MV, Verhulst EC, Zwahlen RD, Beukeboom LW, van de Zande L. 2012. DNA methylation plays a crucial role during early Nasonia development. Insect Molecular Biology 21:129-138. DOI: 10.1111/j.1365-2583.2011.01121.x 


\section{Figure 1 (on next page)}

Figure 1

Schematic of the workflow to produce Nasonia Rearing Media (NRM). Red boxes indicate steps present in NRMv1 but eliminated in NRMv2. Blue boxes indicate steps present in both procedures. Pictures to the right show the visual progression from S. bullata pupae to final NRM product. L, lipid layer; P, protein layer; S, sediment layer. 


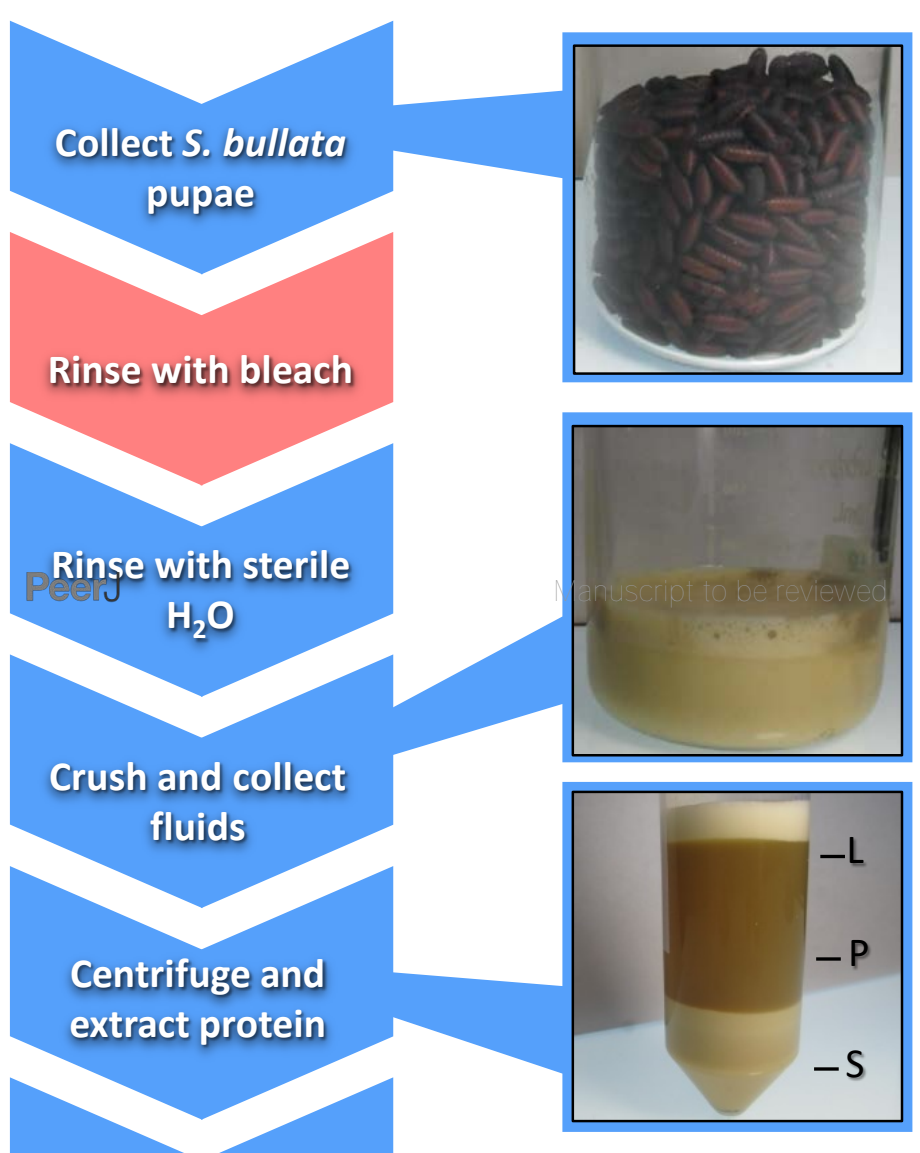

Add Schneider's Drosophila media
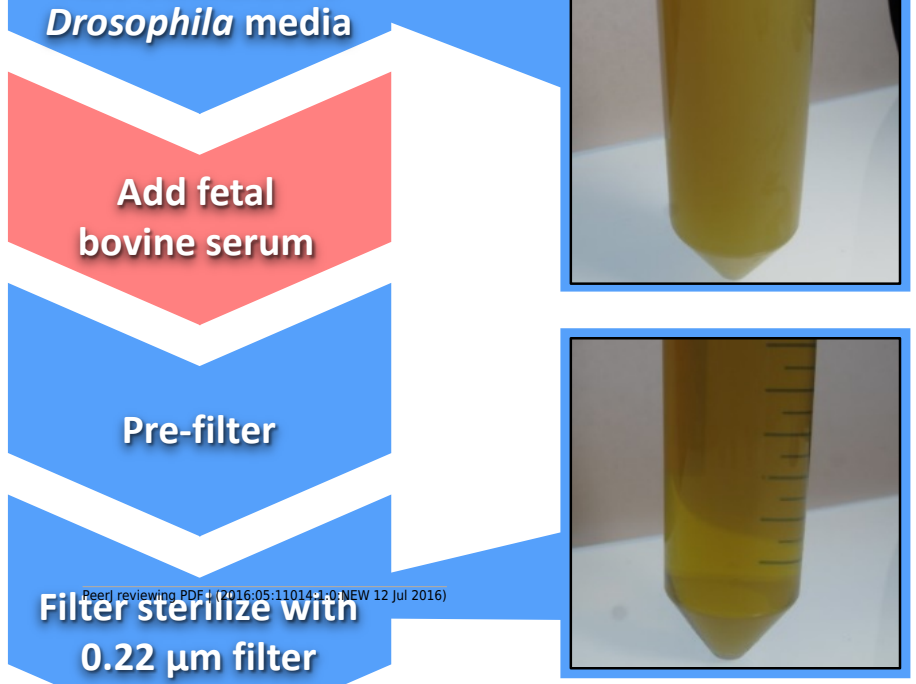

$0.22 \mu \mathrm{m}$ filter

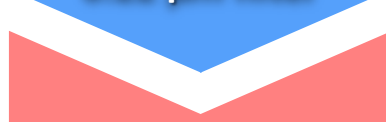

Add antibiotics

Store at $4^{\circ} \mathrm{C}$ until use 


\section{Figure 2 (on next page)}

Figure 2

Comparison of Nasonia germ-free larval development on NRMv1 and NRMv2. (A) The number of living Nasonia vitripennis in transwells on Days 3, 6, and 14. There are no statistically significant differences in larval survival on NRMv1 and NRMv2. (B) Equivalent larval lengths measured from anterior to posterior end in $\mathrm{mm}$. (C-F) Visual comparison of larvae reared on NRMv1 and NRMv2 on Days 6 and 9. Vertical bars with caps represent standard deviation from the mean. 


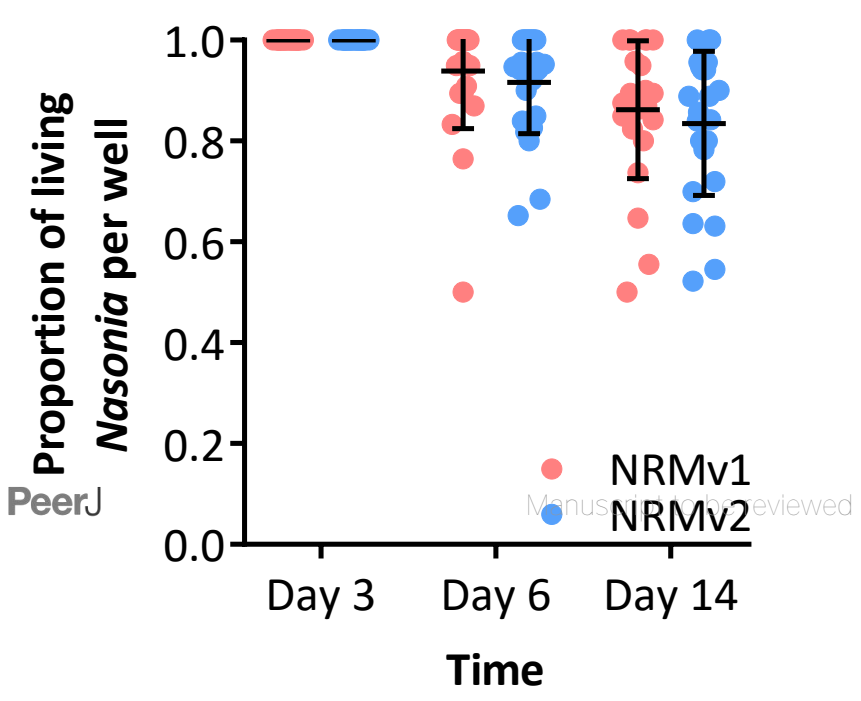

B
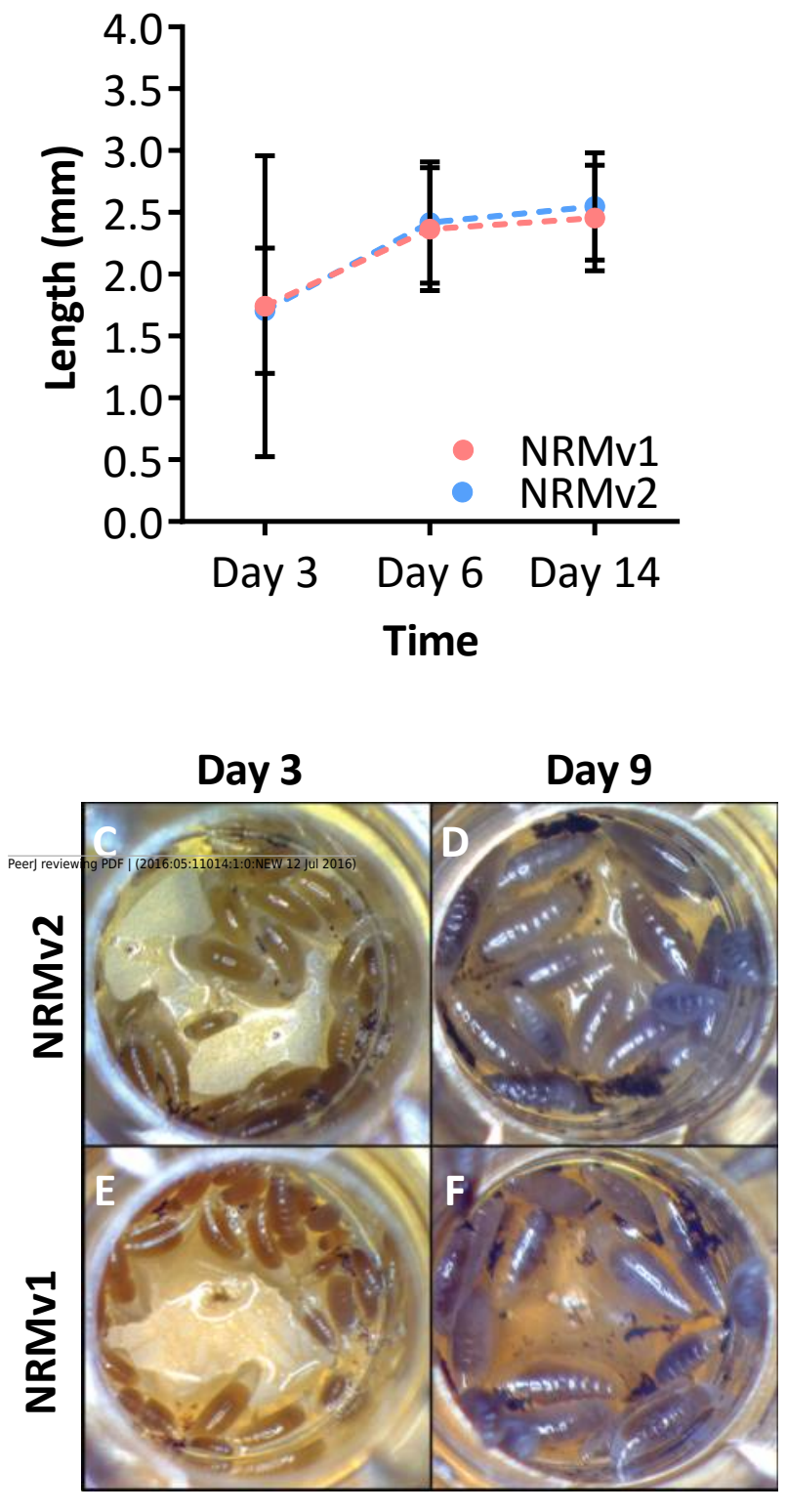
Figure 3 (on next page)

Figure 3

Survival and size of Nasonia germ-free adult males. (A) Comparison of the number of transwells producing adults between NRMv1 and NRMv2. (B) Proportion of larval to adult survival in each transwell is determined as follows: (the number of larvae on Day 3 - the number of larvae and pupae remaining on Day 20) $\div$ the number of larvae on Day 3. (C) Adult head widths from germ-free males reared on NRMv2 and males reared conventionally. Larval to adult adult survival was statistically different between the two media (MannWhitney $\mathrm{U}, \mathrm{P}$-value $=0.001$ ). All other measures were not significant with $\alpha=0.05$. Vertical bars with caps represent standard deviation from the mean. 
A

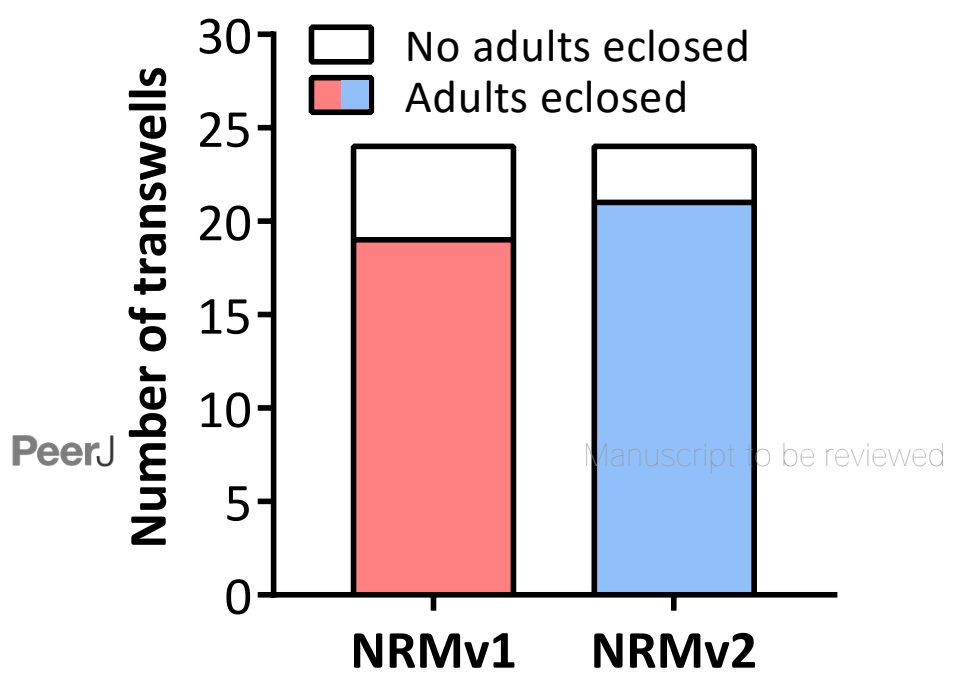

B

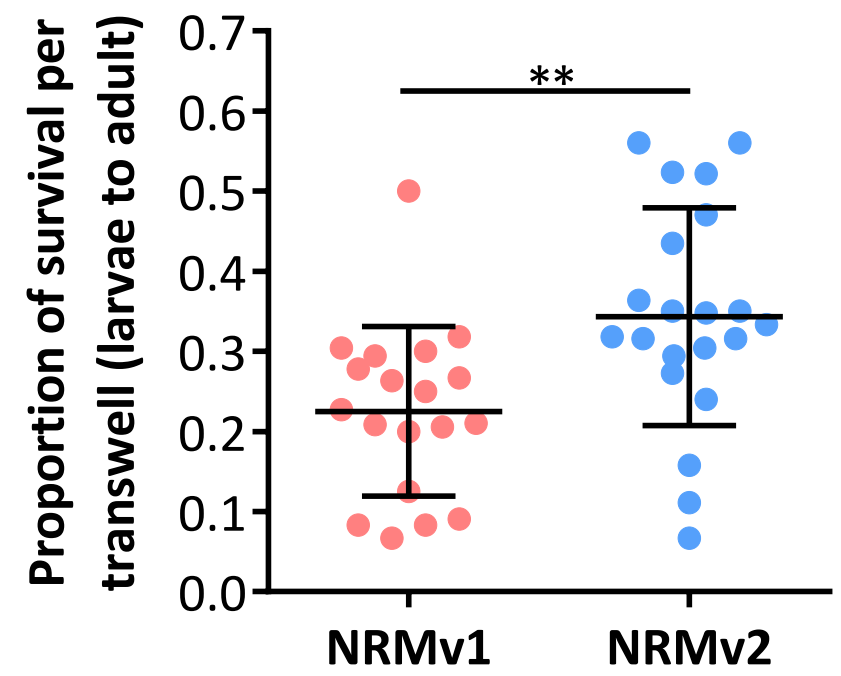

C

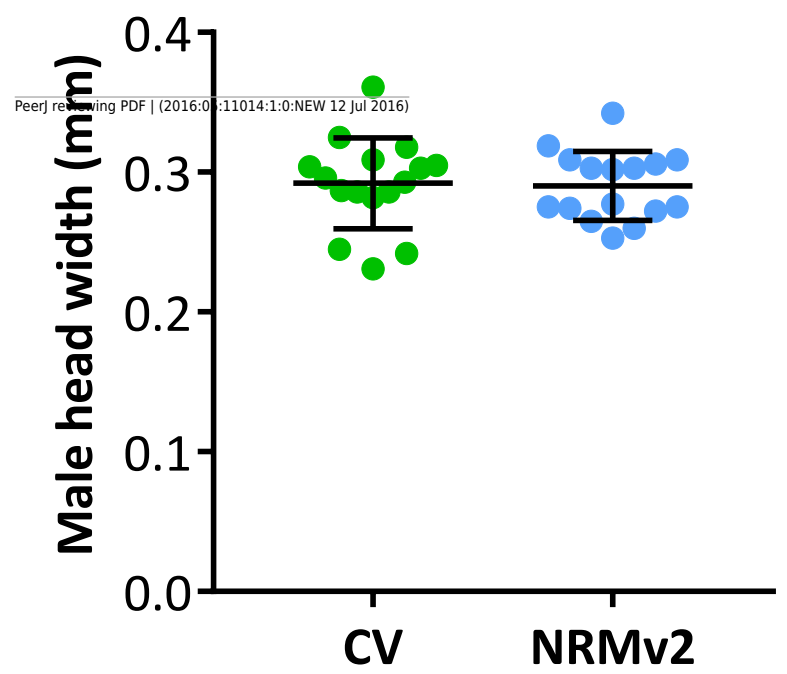

